\title{
Erratum
}

\section{Global uniqueness for an inverse boundary value problem arising in elasticity}

\section{Gen Nakamura ${ }^{1}$, Gunther Uhlmann ${ }^{2}$}

1 Department of Mathematics, Hokkaido University, Sapporo, Hokkaido 060-0810, Japan

2 Department of Mathematics, University of Washington, Seattle, WA 98195, USA

Invent. math. 118, 457-474 (1994)

Oblatum 2-V-2002 \& 7-X-2002

Published online: 17 February 2003 - (c) Springer-Verlag 2003

The proof of Theorem 0.7 in [NU1] is incorrect. Using the same method of proof in [NU1] we can show Theorem 1, below. Unfortunately, we have not been able to prove the global result stated in [NU1].

We now state the corrected result. Let $\Omega$ be a bounded domain in $\boldsymbol{R}^{3}$ with smooth boundary $\partial \Omega$ and let

$$
\begin{aligned}
L u: & =(\lambda+\mu) \nabla(\nabla \cdot u)+\mu \Delta u+(\nabla \cdot u) \nabla \lambda+\left(\nabla u+{ }^{t}(\nabla u)\right) \nabla \mu \\
& =0 \text { in } \Omega
\end{aligned}
$$

be the isotropic elasticity system with Lamé moduli $\lambda, \mu \in C^{\infty}(\bar{\Omega})$ satisfying the strong convexity condition

$$
\mu>0,3 \lambda+2 \mu>0 \text { on } \bar{\Omega} .
$$

Define the Dirichlet to Neumann map $\Lambda_{\lambda, \mu}: C^{\infty}(\bar{\Omega}) \longrightarrow C^{\infty}(\bar{\Omega})$ by

$$
\Lambda_{\lambda, \mu} f:=\left.\sigma(u(f)) \nu\right|_{\partial \Omega},
$$

where $u=u(f) \in C^{\infty}(\bar{\Omega})$ is the solution to

$$
\left\{\begin{array}{l}
L u=0 \text { in } \Omega \\
\left.u\right|_{\partial \Omega}=f,
\end{array}\right.
$$

where $v$ is the outer unit normal vector of $\partial \Omega$ and $\sigma(u)$ is the stress tensor given by

$$
\sigma(u):=\lambda(\operatorname{trace} \nabla u) I+2 \mu \varepsilon(u),
$$


with strain tensor

$$
\varepsilon(u):=\frac{1}{2}\left(\nabla u+{ }^{t}(\nabla u)\right)
$$

Theorem 0.7 in [NU1] holds under additional assumption $\left\|\nabla \mu_{i}\right\|_{C^{m}(\bar{\Omega})}<$ $\varepsilon(i=1,2)$ with some $0<\varepsilon \ll 1$ and $m \in N$. That is we have the following theorem.

Theorem 1 Let $\lambda_{i}, \mu_{i} \in C^{\infty}(\bar{\Omega})(i=1,2)$ be the Lamé moduli satisfying the strong convexity condition. Then, there exist $\varepsilon>0$ and $m \in N$ such that if $\left\|\nabla \mu_{i}\right\|_{C^{m}(\bar{\Omega})}<\varepsilon(i=1,2)$ and $\Lambda_{\lambda_{1}, \mu_{1}}=\Lambda_{\lambda_{2}, \mu_{2}}$, we have $\lambda_{1}=\lambda_{2}$, $\mu_{1}=\mu_{2}$ on $\bar{\Omega}$.

The proof of Theorem 1 follows the general outline of the paper [NU1]. The full details are in [NU2]. Namely we first reduce the second order system of isotropic elasticity to a first order system perturbation of the Laplacian. It is more convenient, as already indicated in [U], to use the reduction of [C] and [An] rather than the one used in [NU1].

The key step in the construction of the exponentially growing solutions (also called complex geometrical optics solutions) is the intertwining property, Theorem 1.23 of [NU1]. The proof of this result goes through with some modifications. See [NU3] for the full details. The main problem in Lemma 1.35 in [NU1] is that we cannot solve in general the initial value problem for the first order system

$$
H_{q_{\zeta}}\left(A_{\zeta, 2}^{(0)}\right)+\psi_{1}\left(s^{-1} \xi_{1}\right) \psi_{2}\left(s^{-1} \xi^{\prime}\right) \sigma\left(N_{\zeta}^{(0)}\right)\left(A_{\zeta, 2}^{(0)}\right)=0 .
$$

We can just solve (1) with $\left(A_{\zeta, 2}^{(0)}\right)$ invertible for large $\zeta$. We use throughout the notation of [NU1]. The method of proof proceeds as in [NU1] by reducing (7) to solve a system of the form

$$
\bar{\partial} A+N A=0 \text { in } \boldsymbol{R}^{2}
$$

depending on parameters. This is straightforward to solve for scalar equations since this is a particular case of a pseudoanalytic equation and all the solutions of (8) can be written in the form of a product of a non-zero function and an holomorphic function. The case of systems is more complicated. Recently Eskin [E] proved that we can find solutions of (8) with $A$ invertible for general systems. We gave an alternative proof of the existence of solutions of (8) in [NU3].

When replacing the exponentially growing solutions constructed in the identity (0.10) of [NU1] we get a pseudodifferential equation rather than a PDE acting on the difference of the Lamé parameters as claimed in [NU1]. We thank G. Eskin and J. Ralston for pointing this to us. We can conclude that we can uniquely identify the Lamé parameters if $\mu$ is a-priori close to a constant. 


\section{References}

[An] Ang, D.D., Ikehata, M., Trong, D.D., Yamamoto, M.: Unique continuation for a stationary isotropic Lamé system with variable coefficients. Comm. Partial Differ. Equations 23, 371-385 (1998)

[C] Chelmiński, K.: The principle of limiting absorption in elasticity. Bull. Pol. Acad. Sci., Math. 41, 19-30 (1993)

[E] Eskin, G.: Global uniqueness in the inverse scattering problem for the Schrödinger operator with external Yang-Mills potentials. Commun. Math. Phys. 222, 503-531 (2001)

[NU1] Nakamura, G., Uhlmann, G.: Global uniqueness for an inverse boundary problem arising in elasticity. Invent. Math. 118, 457-474 (1994)

[NU2] Nakamura, G., Uhlmann, G.: Application of the intertwining property to an inverse boundary value problem in elasticity. To appear CBMS lectures of G. Uhlmann: "Complex geometrical optics and inverse problems". To be published by SIAM

[NU3] Nakamura, G., Uhlmann, G.: Asymptotic methods in inverse problems-complex geometrical optics solutions. In: S.I. Kabanikhin, V.G. Romanov (eds), Ill-Posed and Inverse Problems, 287-320. VSP, The Netherlands 2002

[U] Uhlmann, G.: Developments in inverse problems since Calderón's foundational paper. Harmonic Analysis and Partial Differential Equations, 295-345 (Essays in Honor of Alberto P. Calderón). Chicago: University of Chicago Press 1999 